\title{
Tendon transfer of pronator teres to flexor pollicis longus to restore thumb flexion in incomplete tetraplegia
}

\author{
Nathan P. Jarrett ${ }^{1}$, Gregory A. Merrell, MD² \\ ${ }^{1}$ Medical Student, Indiana University School of Medicine, ${ }^{2}$ Attending Surgeon, \\ Indiana Hand to Shoulder Center
}

Background and Hypothesis: Incomplete tetraplegia creates immense barriers to autonomy for individuals with spinal cord injuries. These patients may retain control of some forearm extensors, but use of flexors is largely eliminated, affecting many movements necessary for daily activities. Although tendon transfers using brachioradialis and other muscles to restore hand function are standard practice, pronator teres has not been extensively studied as a donor for flexors. The purpose of this study is to quantify forearm pronation capability preand post-tendon transfer of pronator teres to flexor pollicis longus in a cadaver model. We hypothesize that tendon transfer will make thumb flexion possible, while preserving forearm pronation against gravity at a minimum.

Experimental Design: Five cadaver arms were evaluated for pronation capability against gravity before and after tendon transfer. In both stages, the arms were also assessed for the pronation forces produced at the wrist when pulling pronator teres with 25,50 , and $75 \mathrm{~N}$ of force. With each force, the arms were tested in full supination and neutral position.

Results: All five arms were capable of pronating against gravity before and after the tendon transfer. Following the transfer, pronation force decreased, but the difference was not statistically significant.

Conclusion and Potential Impact: Pronator teres to flexor pollicis longus tendon transfer produces thumb flexion while retaining the forearm's ability to pronate. Used in conjunction with well-established donors, such as brachioradialis, pronator teres' expendability could offer an additional motor unit for restoring hand function in tetraplegic patients. 